\title{
Size distribution and electroluminescence of self-assembled Ge dots
}

\author{
L. Vescan, ${ }^{\text {a) }}$ T. Stoica, ${ }^{\text {b) }}$ O. Chretien, M. Goryll, E. Mateeva, ${ }^{c}$ and A. Mück \\ Institut für Schicht- und Ionentechnik, Forschungszentrum Jülich GmbH D-52425 Jülich, Germany
}

(Received 1 November 1999; accepted for publication 9 February 2000)

\begin{abstract}
In this article we study the electroluminescence of $p-i-n$ diode structures with Ge dots consisting of coherent three-dimensional small (pyramids) and larger (dome) islands. The Ge dots are formed through strain-induced islanding. The diode structures, including one layer with Ge dots, were deposited on Si mesas with variable areas in order to study the influence of limited area deposition on self-assembling. It was observed that the reduction of deposited area improves island uniformity. The combined analysis of island distribution and electroluminescence spectra has lead to the conclusion that domes in small diodes have a smaller Si content or are less relaxed than domes in larger diodes. The diodes are found to emit up to room temperature near the optical communication wavelength of 1.3 microns. (C) 2000 American Institute of Physics. [S0021-8979(00)04610-7]
\end{abstract}

\section{INTRODUCTION}

For low-cost components in the fiber-optic communication wavelength, silicon-based materials are of great interest due to the possibility of their integration with Si technology. ${ }^{1}$ To overcome the problem of the indirect character of SiGe, one is looking for ways of increasing radiative recombination and reducing nonradiative recombination. One possible approach involves the spatial localization of the injected carriers in three-dimensional (3D) islands. The variation in island size leads to a broadening of spectral emission peaks, therefore, the success of this approach relies on the possibility to control the size, shape, and uniformity of selfassembled islands.

Light emitting diodes with $\mathrm{SiGe}$ islands were first realized in $1995 .^{2}$ The emission was near $\lambda=1.3 \mu \mathrm{m}$ and persisted up to $200 \mathrm{~K}$. Since then, emission was improved by more than 100 times using structures with nominal pure Ge. ${ }^{3,4}$ This was due to the increased valence band offset of the $\mathrm{Ge} / \mathrm{Si}$ heterostructure. The size distribution and the number of island layers was varied and it was found that all these diodes emit up to $300 \mathrm{~K}$ in the range of 1.3-1.4 $\mu \mathrm{m}$. Simulations performed on diodes with thick SiGe layers revealed, in addition, the influence of the interface layer on emission intensity. ${ }^{5}$ The highest emission of diodes with islands achieved so far is from a structure with ten stacked layers with islands. ${ }^{3,4}$ However, by stacking the island layers the size and shape of islands changes. ${ }^{6-8}$ The influence of this effect on the electroluminescence is not yet known. Even the influence of the island distribution on the electroluminescence of a single layer is not well known.

The purpose of this article is to analyze the electroluminescence (EL) of single-island-layer $p-i$ - $n$ diodes. An essential parameter is the diode area as the growth of the whole structure was performed under conditions of selective epitax-

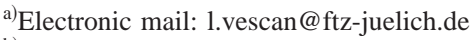

b) Also at: Institutul National de Fizica Materialelor, R76900 Bucharest, MG7, Romania.

c) Also at: Dept. of Metallurgical and Materials Engineering, Colorado School of Mines, Golden, Colorado.
}

ial growth in the form of mesas. It was recently shown that the nucleation of islands on $\mathrm{Si}(001)$ is influenced by the presence of other crystallographic planes. ${ }^{9,10}$ If the mesa size is of the order of the diffusion length of Ge adatoms and if the total coverage is kept below a certain value, then islands nucleate only along mesa edges on $\left\{\begin{array}{lll}1 & 0 & 12\end{array}\right\}$ facets without nucleation on the (001) plane. A surface diffusion length of Ge of the order of $100 \mu \mathrm{m}$ at $700^{\circ} \mathrm{C}$ was evaluated.

The present investigation was performed with a coverage high enough to induce nucleation also on the (001) plane of the mesas. The deposition on limited areas with sizes of the order of the diffusion length was observed to change the size distribution. A narrowing of the size distribution occurs when the area is reduced from a large area $\left(1 \mathrm{~cm}^{2}\right)$ down to squares of $50 \mu \mathrm{m}$ size. The analysis of electroluminescence spectra revealed the influence of the mesa area on the microstructure of islands. It was found that single diodes as well as arrays emit up to room temperature, the highest intensity being detected from small area diodes. Experimental details are presented in Sec. II, the diode structure is discussed in Sec. III, a review of our own results on 3D island distribution is presented in Sec. IV, and the electroluminescence data are presented and analyzed in Sec. V.

\section{EXPERIMENTAL DETAILS}

The epitaxy was performed in a low pressure chemical vapor deposition system (LPCVD) at 0.12 Torr. $^{7}$ Source gases are $\mathrm{SiCl}_{2} \mathrm{H}_{2}$ and $\mathrm{GeH}_{4}(10 \%$ in $\mathrm{He})$ while $\mathrm{H}_{2}$ is used as a carrier gas. Doping gases are $\mathrm{B}_{2} \mathrm{H}_{6}\left(20 \mathrm{ppm}\right.$ in $\left.\mathrm{H}_{2}\right)$ and $\mathrm{PH}_{3}\left(100 \mathrm{ppm}\right.$ in $\left.\mathrm{H}_{2}\right)$. The deposition was performed on $n^{+}$ $\mathrm{Si}(001)$ substrates (doping concentration $10^{19} \mathrm{~cm}^{-3}$ ) with $300 \mathrm{~nm}$ thermal oxide patterned with square holes with sizes from $4 \mu \mathrm{m}$ up to $1 \mathrm{~mm}$. Before epitaxy the wafers were cleaned by RCA cleaning, while the protective $2 \mathrm{~nm}$ oxide was removed in situ at $950{ }^{\circ} \mathrm{C}$ for 10 min under $\mathrm{H}_{2}$ flow. The epitaxial growth is selective and the growth rate and Ge content of $\mathrm{Si}_{1-x} \mathrm{Ge}_{x}$ on $\mathrm{Si}$ (001) do not depend on mesa size down to $0.5-1 \mu \mathrm{m} .{ }^{11}$ This implies that independent of mesa area, all diode structures analyzed here have the same layer 
TABLE I. Layer sequence of the LPCVD grown island diodes (No. 1445).

\begin{tabular}{|c|c|c|c|c|c|}
\hline & Layer & $\begin{array}{c}\text { Deposition } \\
\text { temperature } \\
{ }^{\circ} \mathrm{C}\end{array}$ & $\begin{array}{c}\text { Thickness } \\
/ \mathrm{nm}\end{array}$ & $\begin{array}{l}\text { Doping } \\
/ \mathrm{cm}^{-3}\end{array}$ & $\begin{array}{l}\text { Nominal } \\
\text { Ge content }\end{array}$ \\
\hline \multirow[t]{3}{*}{$n+$} & $n^{+}$Si buffer & 800 & 20 & $1 \times 10^{18}$ & 0 \\
\hline & (n-Si buffer & 800 & 280 & $6 \times 10^{17}$ & 0 \\
\hline & $n$-Si buffer & 700 & 5 & $6 \times 10^{17}$ & 0 \\
\hline \multirow[t]{2}{*}{$i$} & Ge layer & 700 & 1.3 & $?$ & 1 \\
\hline & $n$-SiGe spacer & 700 & 28 & $4 \times 10^{17}$ & 0.02 \\
\hline \multirow[t]{2}{*}{$p+$} & $p^{+} \mathrm{SiGe}$ & 700 & 122 & $2 \times 10^{19}$ & 0.02 \\
\hline & $p^{+} \mathrm{Si}$ & 700 & 4 & $2 \times 10^{19}$ & 0 \\
\hline
\end{tabular}

thickness. The layer thickness, composition, and doping were determined by Rutherford backscattering spectrometry and secondary ion mass spectrometry.

Table I shows the layer sequence. The $p-i-n$ diodes contain an " $i$ " region with an average $n$-type doping of 5 $\times 10^{17} \mathrm{~cm}^{-3}$. The growth parameters for the $\mathrm{Ge}$ islands were: $p_{\mathrm{GeH} 4}=5 \times 10^{-4}$ Torr for a flow rate of $\mathrm{GeH}_{4}$ of 10 sccm, average growth rate $2.6 \mathrm{~nm} / \mathrm{min}$, and deposition time $30 \mathrm{~s}$. This resulted in an average layer thickness for Ge of 1.3 $\mathrm{nm}$ (approximately $9 \mathrm{ML}$ ). After the Ge deposition an $1 \mathrm{~min}$ annealing at $700{ }^{\circ} \mathrm{C}$ followed, before capping. The $\mathrm{SiGe}$ spacer and the $p^{+}$SiGe layer contain $2 \%$ Ge. Adding $2 \% \mathrm{Ge}$ increases the growth rate approximately 2 times and thus significantly decreases deposition time and intermixing of $\mathrm{Ge}$ from the islands and $\mathrm{Si}$.

The surface topography was investigated with a Digital Instruments Nanoscope IIIa atomic force microscope (AFM) in tapping mode and with a scanning electron microscope

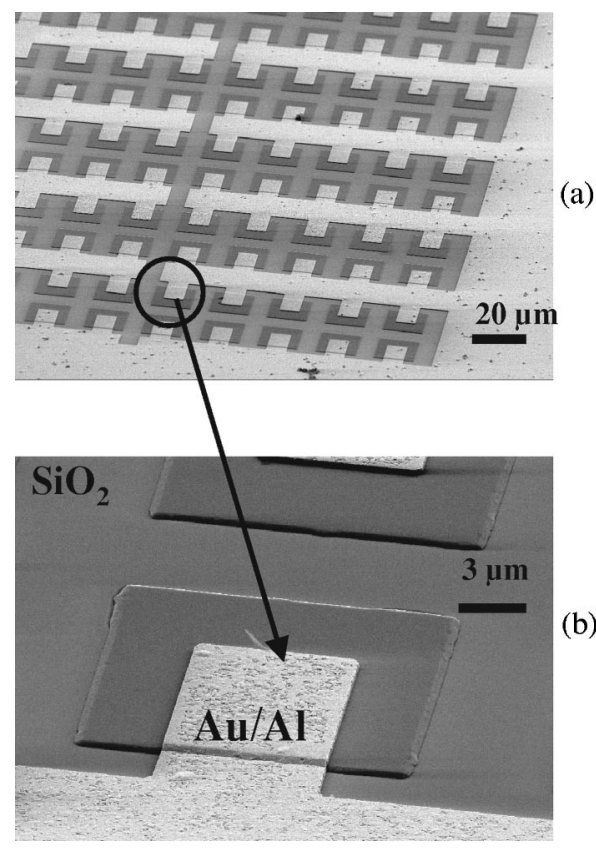

FIG. 1. SEM pictures showing the mesa diodes. Tilt angle $60^{\circ}$. (a) array of 100 diodes, each diode with an area of $24 \times 24 \mu \mathrm{m}^{2}$ and (b) enlarged picture of one diode.
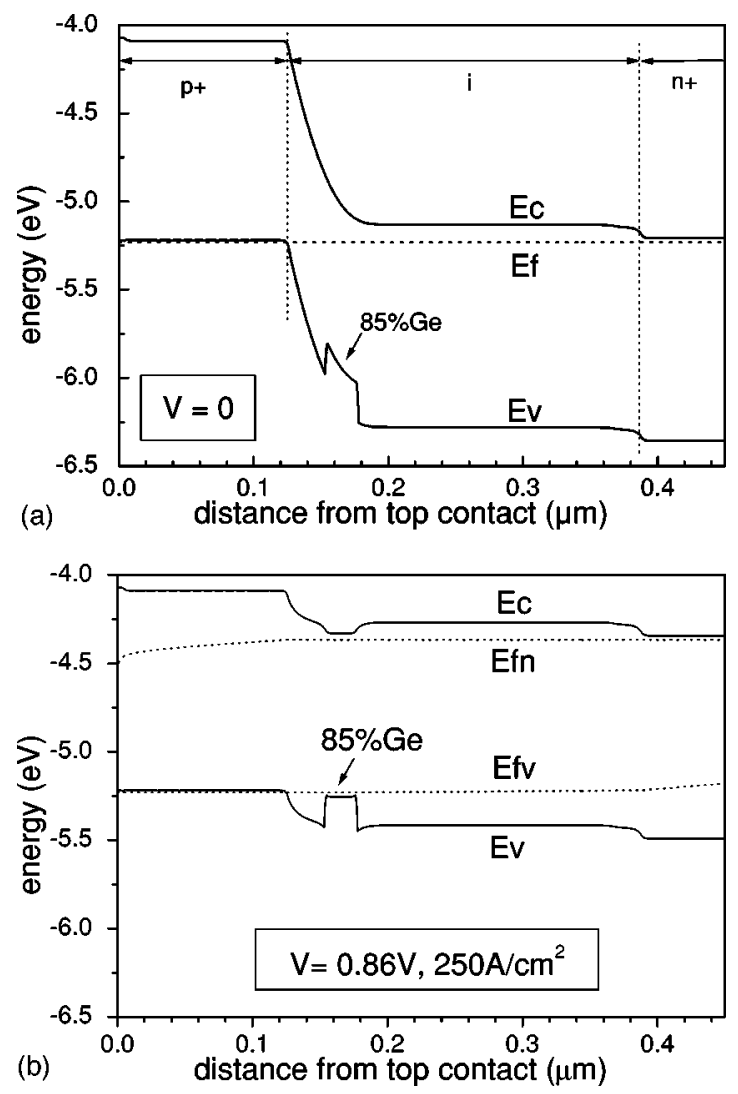

FIG. 2. Simulated band diagram of a diode: (a) at zero bias and (b) forward bias. A thickness of $25 \mathrm{~nm}$ for the dots and a concentration of $15 \% \mathrm{Si}$ in the dots was assumed.

(SEM). Cross-sectional transmission electron microscopy (XTEM) was performed using a Philips CM200 microscope at $200 \mathrm{KV}$. The XTEM samples were prepared by tripod polishing followed by brief ion milling. The EL was measured with a Fourier transform spectrometer equipped with a cooled Ge detector with the diodes fixed in a He cryostat.

\section{DIODE CHARACTERISTICS}

Figure 1(a) displays a scanning electron micrograph of an array of 100 diodes of $24 \mu \mathrm{m}$ square mesas connected in parallel and Fig. 1(b) shows an enlarged picture of one mesa. The diodes have a front emitting structure with an ohmic contact of evaporated Al/Au. Usually an annealing at $350{ }^{\circ} \mathrm{C}$ for $5 \mathrm{~min}$ is performed to achieve an ohmic front contact. The back contact was Ga-In. All diodes in Fig. 1 are connected in parallel.

The band diagram of the diode was calculated with the SIMWIN program (Stanford University) using the data for layer thickness and doping levels from Rutherford backscattering spectrometry and secondary ion mass spectrometry (see also Table I). The island was simulated by a quantum well with a thickness of $25 \mathrm{~nm}$ (typical height of islands) and a relaxed band gap with $85 \%$ Ge. Figure 2 shows a simulation for zero bias and one for $V_{f}=0.86 \mathrm{~V}$. Figure 2(b) shows that biasing the diode in forward, holes are expected to be captured in islands and therefore to recombine in a certain proportion radiatively. 

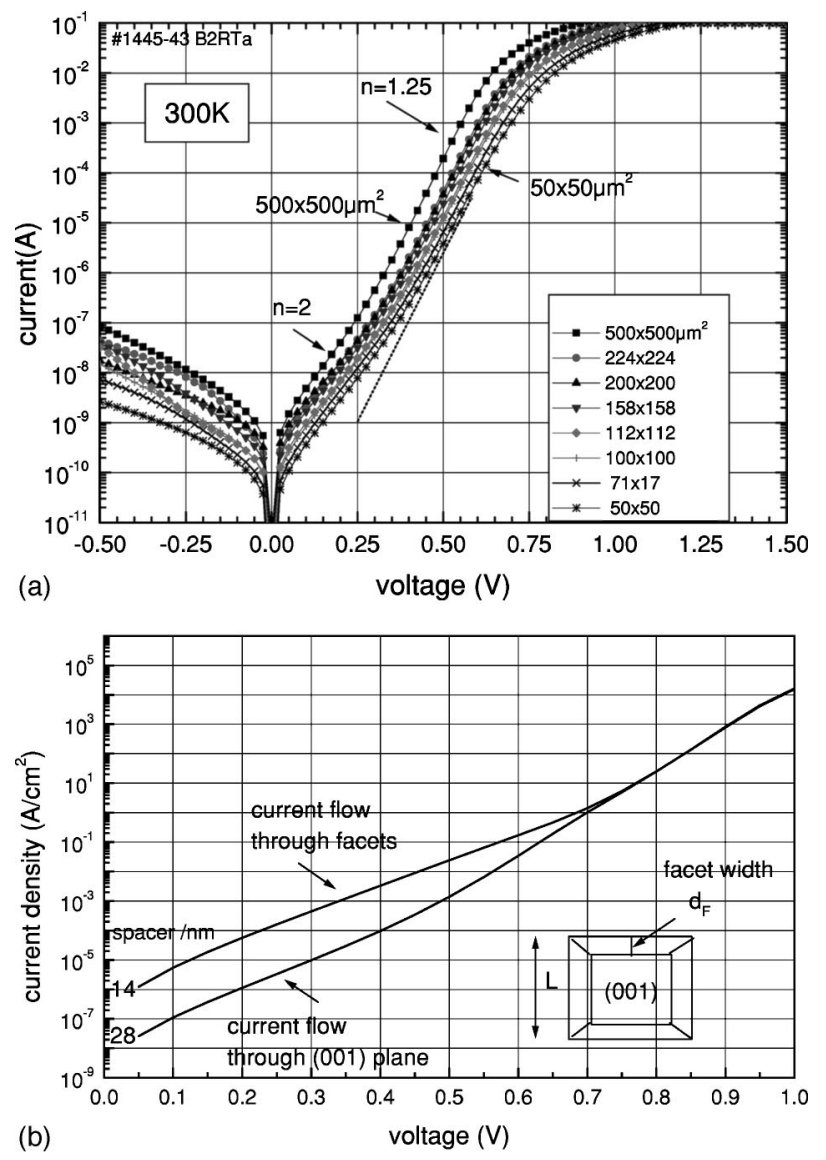

FIG. 3. Current-voltage characteristics. (a) a set of curves from single mesa diodes with different areas measured at $300 \mathrm{~K}$. The dotted line shows where the $I-V$ curve for the $50 \times 50 \mu \mathrm{m}^{2}$ diode should be if there would have been a $L^{2}$ dependence. (b) simulated curves based on the doping and thickness data of Table I. The thickness of the $n$-SiGe spacer was varied.

Current-voltage $(I-V)$ characteristics measured at 300 $\mathrm{K}$ for single diodes with square areas of dimension $L$ are shown in Fig. 3(a). Above $\sim 0.7 \mathrm{~V}$ the contact resistance limits the current. At lower voltages the current scales, not as expected, with the diode area, but a dependence $I \sim L^{\alpha}$ with $1<\alpha<2$ is observed. If the current would scale with $L^{2}$, then for instance, for a diode with $L=50 \mu \mathrm{m}$ the current should have been $10^{-9} \mathrm{~A}$ at $0.25 \mathrm{~V}$ and $2.10^{-6} \mathrm{~A}$ at $0.5 \mathrm{~V}$ (see dotted line). The $\alpha<2$ behavior implies either a leakage current at the periphery of the diode or, what we believe in our case, a higher current density through the mesa facets than across the bulk region [see inset of Fig. 3(b)]. The thickness of all layers in the facet region are thinner than along the [001] direction. In an earlier study we have demonstrated that the growth rate of the facets is $0.5-0.7$ times lower than the growth rate along [001] directions. ${ }^{12}$ Thereby we have simulated the $j-V$ characteristics (j-current density) and we found that the spacer thickness (the layer between the island layer and the $p^{+}$contact, see Table I) is a critical parameter. Figure 3(b) shows a curve corresponding to a spacer of 28 $\mathrm{nm}$, which should correspond to the current flow across the (001) part of the mesa. For the current density across the facet region we took half this value (all other parameters were the same) and the extension of the facet was assumed to be $0.3 \mu \mathrm{m}$. We see that at lower voltages $j$ through facets is approximately 100 times higher than $j$ through the (001) part. With this value it is possible to explain the deviation of the scale dependence of the current from $\alpha=2$ to $\alpha<2$. This calculation also shows that in order to avoid higher currents through facets, a spacer thicker than $56 \mathrm{~nm}$ along the [001] direction should be chosen.

At low voltage the ideality factor is $\sim 2$, implying recombination on deep centers in the field region. As the barrier shrinks with forward voltage, recombination in the neutral " $i$ " region becomes more important. The ideality factor tends to $\sim 1.25$ indicating medium recombination with comparable recombination times. ${ }^{13}$

\section{3D ISLAND GROWTH DETAILS}

Before discussing the results on electroluminescence of Ge islands it is meaningful to summarize some of our results on self-assembled growth of Ge. The variation of island size leads to a broadening of the spectral emission peaks, ${ }^{14-16}$ therefore, understanding of the parameters responsible for the size distribution is important. Nucleation of $3 \mathrm{D}$ islands is the result of a transition in growth mode of a layer when the strain energy exceeds a certain value during growth. ${ }^{17}$ During and after this transition the surface migration phenomenon plays an important role. The general observation is that island distribution and island density depend on growth parameters such as temperature, total coverage, growth rate and time, chemical species, faceted substrates, etc. ${ }^{8-10,14-24}$ All of these parameters influence the surface migration and implicitly the island formation.

Recently, Goryll et al. examined the influence of growth rate on the island size distribution for uncapped islands and for coverage of $\sim 0.9 \mathrm{~nm}$, low enough to avoid plastic relaxation of islands. ${ }^{16}$ AFM topography revealed two types of islands for a growth rate higher than $0.4 \mathrm{~nm} / \mathrm{min}$, in agreement with the literature. ${ }^{21,23}$ Small islands (pyramidal cluster $^{17}$ with $\{105\}$ facets) of high density coexist with medium sized islands (multifaceted islands called domes ${ }^{18}$ ) of lower density (see sample No. 1377 in Table II). By AFM, two distinct distributions in height (centerd at 4.5 and $15 \mathrm{~nm}$, respectively) and one broad distribution in diameter (centered approximately at 60 and $90 \mathrm{~nm}$ ) were observed. The transition from a bimodal to a monomodal distribution, observed by AFM but also by photoluminescence of capped samples ${ }^{16}$ at constant Ge coverage, occurred when the growth rate was decreased.

In the present study, the growth of Ge was performed with a relatively high growth rate $(2.6 \mathrm{~nm} / \mathrm{min}=0.3 \mathrm{ML} / \mathrm{s})$, therefore bimodality was expected on a large area. ${ }^{16}$ Beside the growth rate, the annealing and/or deposition time influences the island shape and shape transition. ${ }^{22,23}$ This is summarized in Table II. For a deposition time of $18 \mathrm{~s}$ (No. 1377) pyramids and domes coexist and there are five times more pyramids than domes. Annealing leads to an increase of the size of domes and pyramids (No. 1490) and therefore a strong reduction of their density. By increasing the coverage from 0.9 to $1.3 \mathrm{~nm}$ the density of domes increases, while the density of pyramids decreases. During annealing, Ge ada- 
TABLE II. Influence of deposition and annealing time on island distribution on large areas; $\rho$-density of islands, $D$-diameter, $H$-height, $D$-domes, $P$-pyramids.

\begin{tabular}{cccccccccc}
\hline \hline No. & $\begin{array}{c}\text { Dep. } \\
\text { time/s }\end{array}$ & $\begin{array}{c}\text { Anneal. } \\
\text { time/s }\end{array}$ & $\begin{array}{c}\text { Coverage } \\
/ \mathrm{nm}\end{array}$ & $\begin{array}{c}\rho_{D} \\
/ \mu \mathrm{m}^{-2}\end{array}$ & $\begin{array}{c}\rho_{P} \\
/ \mu \mathrm{m}^{-2}\end{array}$ & $\begin{array}{c}D_{D} \\
/ \mathrm{nm}\end{array}$ & $\begin{array}{c}D_{P} \\
/ \mathrm{nm}\end{array}$ & $\begin{array}{c}H_{D} \\
/ \mathrm{nm}\end{array}$ & $\begin{array}{c}H_{P} \\
/ \mathrm{nm}\end{array}$ \\
\hline 1377 & 18 & 0 & 0.8 & 5 & 27 & 90 & 60 & 15 & 4.5 \\
1490 & 18 & 42 & 0.8 & 3 & 7 & $130-170$ & $60-130$ & 23 & 10 \\
1554 & 30 & 0 & 1.3 & 20 & 3 & 120 & $50-80$ & 24 & 4 \\
1608 & 30 & 60 & 1.2 & 6 & 0.4 & $100-230$ & $60-90$ & $15-48$ & $1-3$ \\
\hline \hline
\end{tabular}

toms have time to diffuse to the more stable domes, increasing their size and reducing the total island density (No. 1608).

The size distribution of islands which corresponds to the $p-i-n$ diodes was evaluated on the uncapped sample No. 1608 , grown with the same growth rate and time as the diode sample (see Table II, III), and Fig. 4). Table III shows that all mesas have a bimodal distribution with a total density of $\sim 10 \mu \mathrm{m}^{-2}$ and with only $10 \%$ pyramids. An interesting result is that the reduction of the deposited area has a beneficial effect on size distribution. This becomes much narrower, both in diameter and height. This is clearly demonstrated in Fig. 4 where AFM scans and histograms for large area and $50 \times 50 \mu \mathrm{m}^{2}$ mesas are compared. While on large areas the diameter and height distributions are broad, on the $50 \times 50$ $\mu \mathrm{m}^{2}$ mesas the distributions are much narrower. For instance big islands of height $31-48 \mathrm{~nm}$ and diameter $180-230 \mathrm{~nm}$ seen on large areas, do not form on the small mesas. To conclude, the $50 \times 50 \mu \mathrm{m}^{2}$ mesas have a higher dome density, smaller dome sizes, and a better uniformity than the large areas. In addition, Table III shows that there is not much difference in the size distribution between the 500 $\times 500$ and $50 \times 50 \mu \mathrm{m}^{2}$ diodes. This can be explained by the fact that all these mesas are of the order of magnitude of the diffusion length of $100 \mu \mathrm{m}$ and that most of the deposited atoms nucleate on the (001) plane, the number of Ge islands nucleated on the facets being 20 times lower.

To realize diodes, capping is necessary. However, the capping is known to change the island shape. ${ }^{6}$ However, it seems that the density of islands and the bimodality (if existent) are preserved during capping. Figure 5 shows a TEM of the diode structure performed on an unpatterned area. All islands are dislocation free and the surface morphology is flat (at least at this magnification), which indicates that after depositing the $\mathrm{Ge}$ islands and during the growth of the $\mathrm{Si}_{0.98} \mathrm{Ge}_{0.02}$ layers, the $\mathrm{Si}$ atoms are preferentially incorporated between the islands, where the lattice parameter is nearer to that of bulk silicon, leading to a planarized surface, as discussed in the literature. ${ }^{7,25-27}$ To summarize, all mesa diodes have a dome density of $10 \mu \mathrm{m}^{-2}$ and a pyramid density of $0.4-1.2 \mu \mathrm{m}^{-2}$.

\section{ELECTROLUMINESCENCE OF 3D ISLANDS}

The temperature dependence of the electroluminescence is shown in Fig. 6. The EL spectra from a single $200 \times 200$ $\mu \mathrm{m}^{2}$ diode are compared with EL from sixteen $50 \times 50 \mu \mathrm{m}^{2}$ diodes measured with the same current (therefore the injected current density is the same). The emission from $\mathrm{Ge}$ islands lies in the range $800-950 \mathrm{meV}$. A redshift of the peaks is observed with increasing temperature. In Fig. 6(c) the curves for $375 \mathrm{~A} \mathrm{~cm}^{-2}$ correspond to the two diodes of Figs. 6(a) and 6(b). Other diode combinations are represented in addition showing a redshift with temperature, too. The dotted lines in Fig. 6(c) show the temperature variation of the band gap for bulk $\mathrm{Ge}_{x} \mathrm{Si}_{1-x}$ for two Ge concentrations (from Braunstein et $_{\text {al. }}{ }^{28}$ ). Comparing the experimental redshift of the electroluminescence of islands with the temperature variation of the band gap one can conclude that the redshift of EL is roughly due to the band gap shrinkage with temperature. For a detailed understanding of the temperature dependence of the ground state transition energy numerical calculations are required.

The current dependence of the EL is shown in Fig. 7. EL spectra of a single square $100 \mu \mathrm{m}$ diode are compared with spectra of an array of $50 \mu \mathrm{m}$ square diodes but with the same total area. We see that the EL intensities are comparable, the current dependence $\left(I_{\mathrm{EL}} \sim I^{m}\right)$ has an exponent $m \sim 1$ and in both cases there is a blueshift of the EL peak with increasing injection current. The EL peak position, corresponding to the diodes of Fig. 7 and of several other diodes, is plotted as a function of injected current in Fig. 8(a). All EL peaks reveal a blueshift with the current. Sample heating is not responsible for this effect, since it would reduce the band gap of $\mathrm{Ge}$

TABLE III. Island distribution for an uncapped sample (No. 1608) grown with the same growth rate and total coverage of $1.3 \mathrm{~nm}$ as the diode sample (No. 1445); $\rho_{D^{-}}$-density of domes, $\rho_{P}$-density of pyramids.

\begin{tabular}{cccccc}
\hline $\begin{array}{c}\text { Pad size } \\
/ \mu \mathrm{m}^{2}\end{array}$ & $\begin{array}{c}\text { Total nr. of } \\
\text { islands in a mesa }\end{array}$ & $\begin{array}{c}\rho_{D} \\
/ \mu \mathrm{m}^{-2}\end{array}$ & $\begin{array}{c}\rho_{P} \\
/ \mu \mathrm{m}^{-2}\end{array}$ & $\begin{array}{c}\text { Diameter of } \\
\text { domes } / \mathrm{nm}\end{array}$ & $\begin{array}{c}\text { Height of } \\
\text { domes } / \mathrm{nm}\end{array}$ \\
\hline$>10^{8}$ & $\gg 10^{8}$ & 6 & 0.4 & $100-230$ & $15-48$ \\
$500 \times 500$ & $270 \times 10^{4}$ & 10 & 0.4 & $110-180$ & $11-31$ \\
$200 \times 200$ & $42 \times 10^{4}$ & 10 & 0.7 & $100-190$ & $11-35$ \\
$100 \times 100$ & $11 \times 10^{4}$ & 10 & 1.2 & $100-175$ & $10-33$ \\
$50 \times 50$ & $2.7 \times 10^{4}$ & 10 & 1.2 & $100-170$ & $12-31$ \\
\hline \hline
\end{tabular}



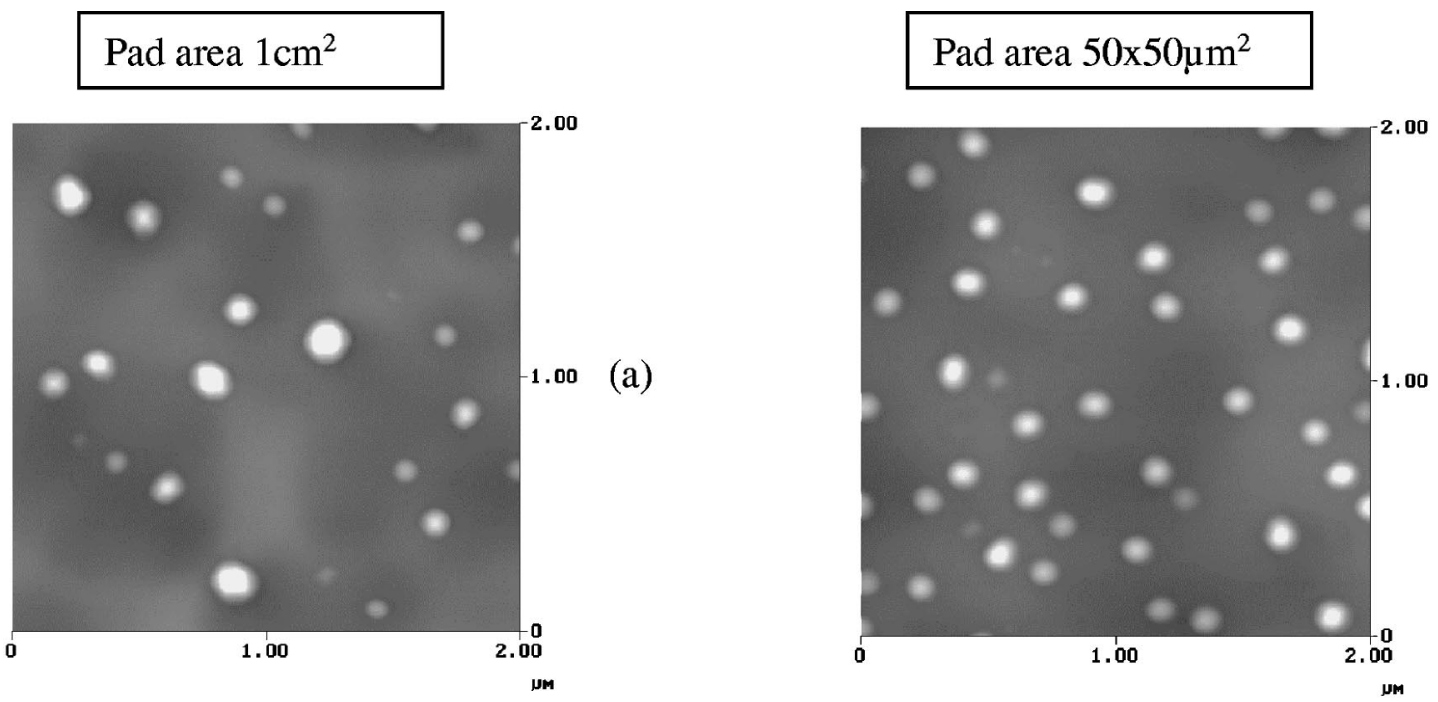

(d)
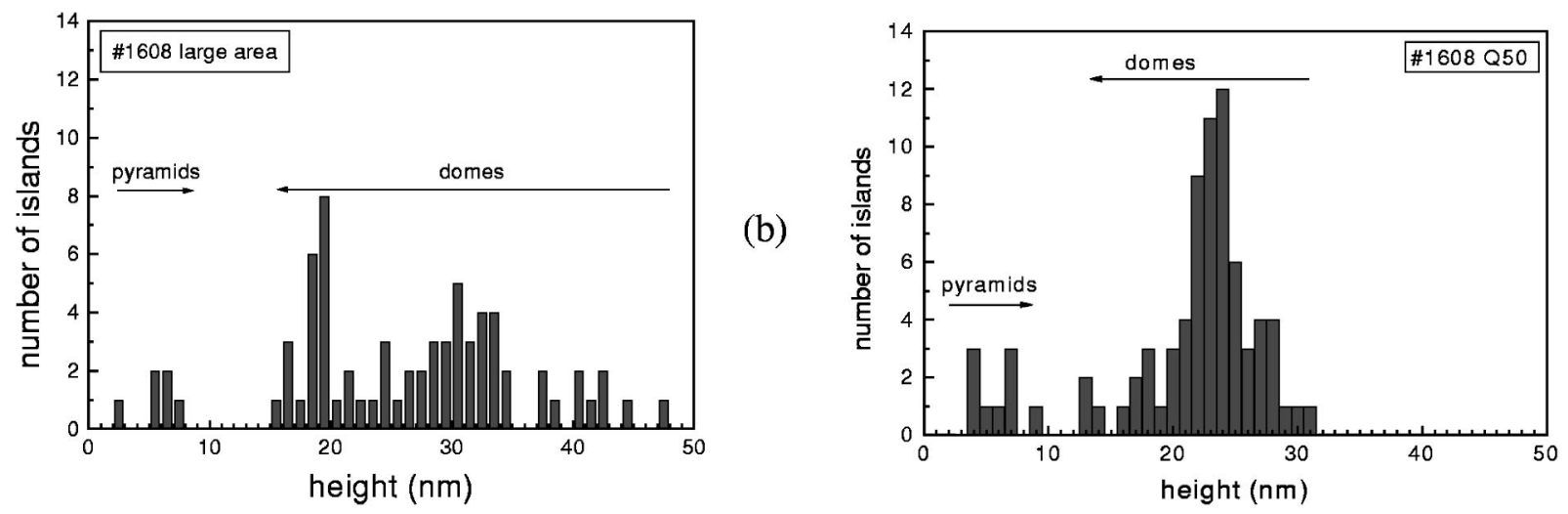

(e)
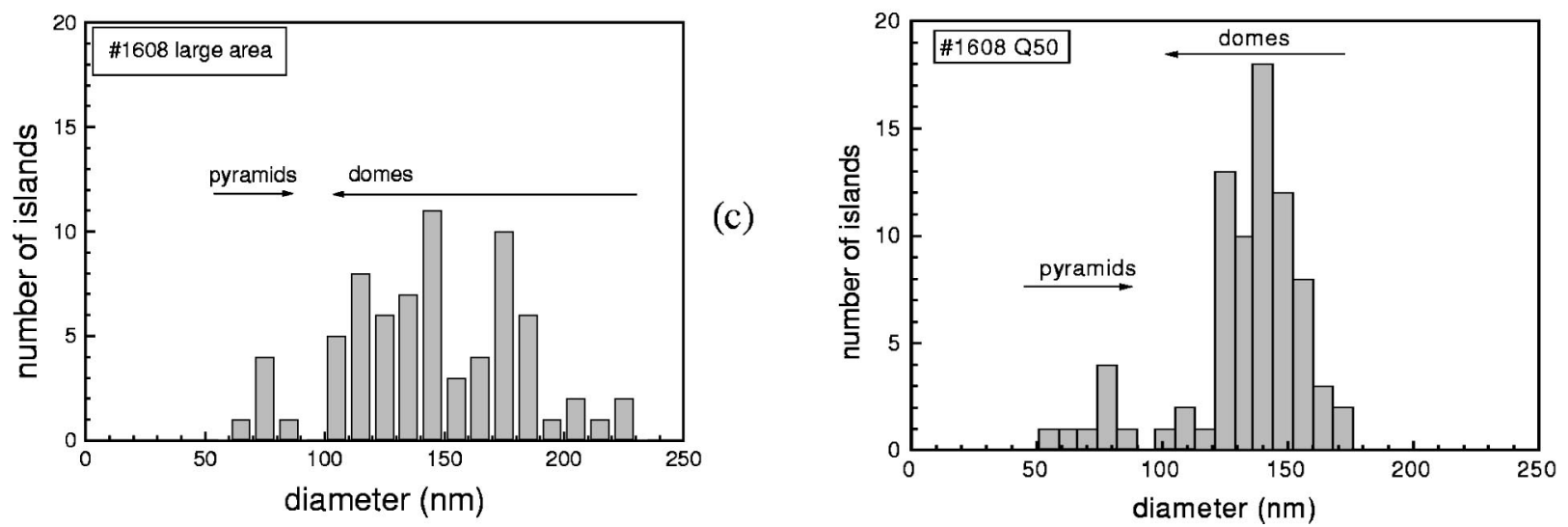

FIG. 4. AFM pictures and size distribution for a large area part of $1 \mathrm{~cm}^{2}(\mathrm{a}, \mathrm{b}, \mathrm{c})$ and a $50 \times 50 \mu \mathrm{m}^{2}$ mesa (d,e,f).

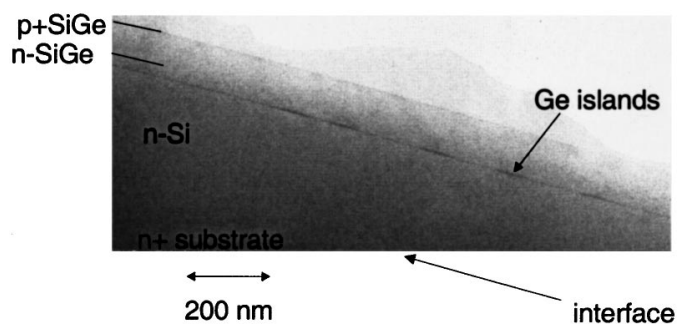

FIG. 5. TEM cross section along a $\langle 110\rangle$ direction on a piece cut from a large area sample. All five islands seen in cross section are dislocation free. and $\mathrm{Si}$ and produce a redshift, as seen above. A spectral peak shift results when the lower energy states from Ge islands become saturated. Because most of the islands have a height above $10 \mathrm{~nm}$ (the height being the quantum dimension) the energy level separation is quite small $(<13 \mathrm{meV})$. Therefore, the peaks from the different energy levels are not resolved and a smooth shift of EL occurs during the band filling. The shift saturates between 860 and $920 \mathrm{meV}$ depending on current and mesa area. But, it seems that this shift is the combined effect of band filling and island distribution. 

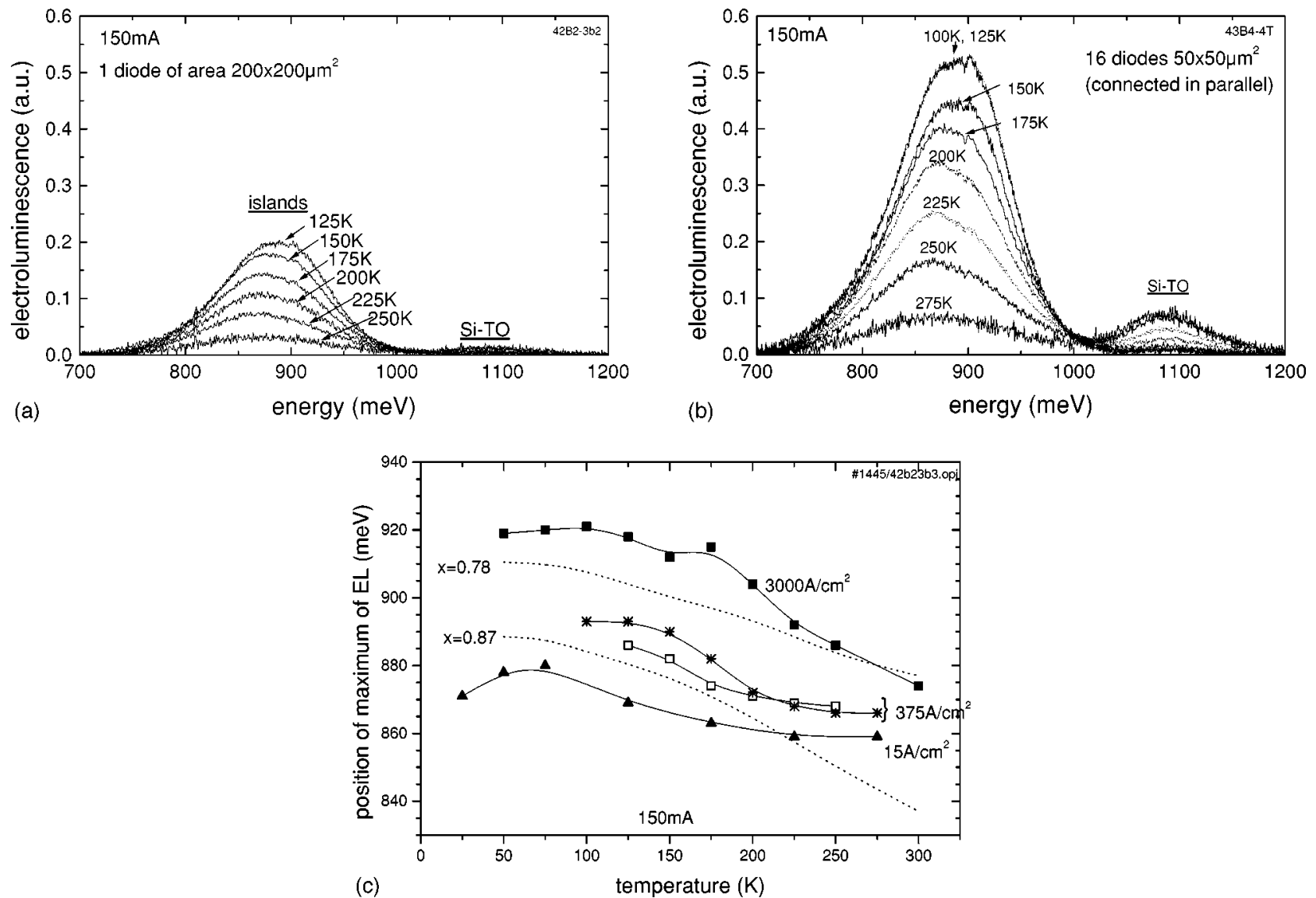

FIG. 6. Temperature dependence of EL measured at $150 \mathrm{~mA}$ (the units for the EL intensity are the same for all spectra, including Figs. 7(a) and 7(b)): (a) spectral distribution of electroluminescence of one diode of area $200 \times 200 \mu \mathrm{m}^{2}$ and (b) array of 16 diodes of area $50 \times 50 \mu \mathrm{m}^{2}$ connected in parallel; (c) shift with temperature of the EL peak maximum for different diodes corresponding to different current densities; the dotted lines represent the variation of the band gap of bulk $\mathrm{Ge}_{x} \mathrm{Si}_{1-x}$ for a given $x$ (from Ref. 28).

To understand this behavior in more detail, the data of Fig. 8(a) were represented in Fig. 8(b) in dependence on the current divided by the total number of islands in each diode (see Table III). This representation reveals a different behavior between small and larger diodes. While the peak position of all diodes larger than $100 \times 100 \mu \mathrm{m}^{2}$ lies on the same curve, the data of the $50 \times 50 \mu \mathrm{m}^{2}$ diodes follow a curve at lower energy, at least below a certain current.

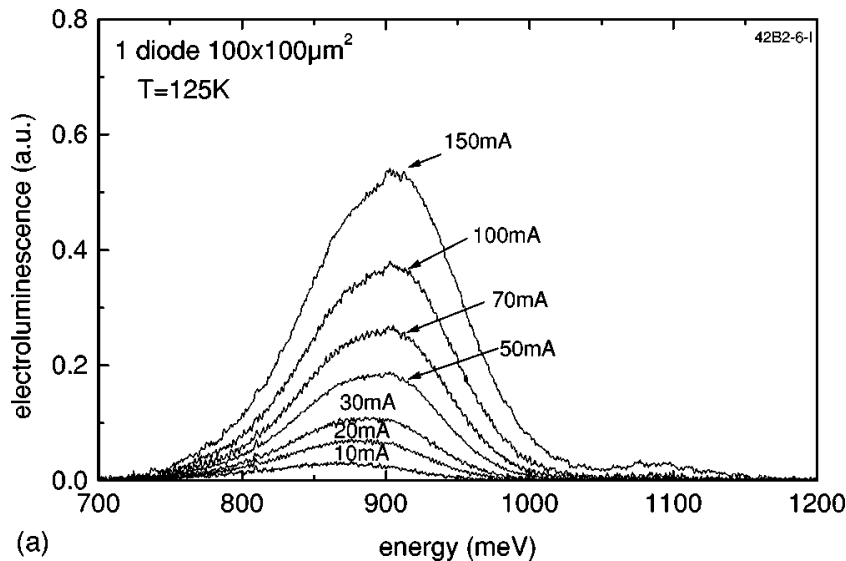

However, the representation in Fig. 8(b) has to be considered critically. The current is not expected to flow only through islands. A part, and probably a remarkable part of it flows between the islands. Therefore, the current through one island must be smaller than the current given in Fig. 8(b). The coverage with islands is quite small $(\sim 20 \%)$ both for the larger diodes as well as for the smaller ones. If we take this into consideration there will be a shift of all curves to lower

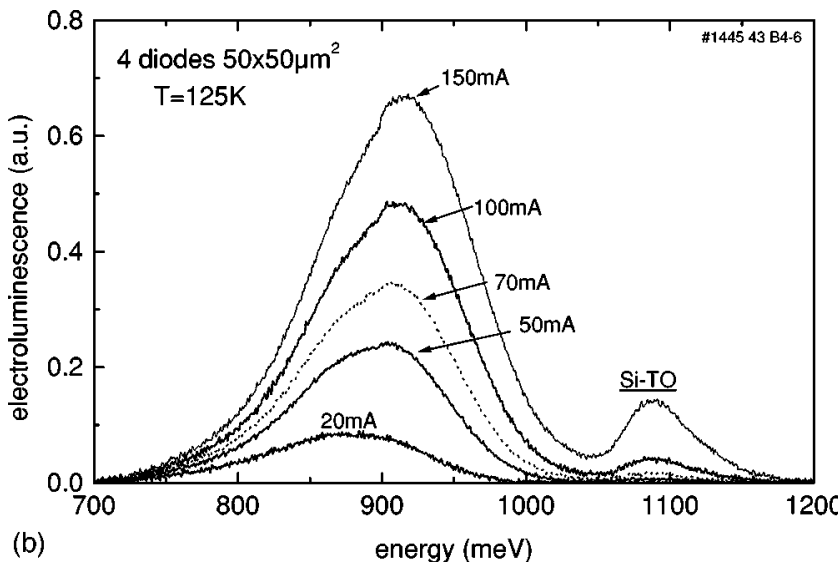

FIG. 7. Spectral distribution of electroluminescence of diodes with the same total area measured at different currents at $125 \mathrm{~K}$. (a) one diode of area $100 \times 100$ $\mu \mathrm{m}^{2}$ and (b) array of four diodes of area $50 \times 50 \mu \mathrm{m}^{2}$ connected in parallel. 

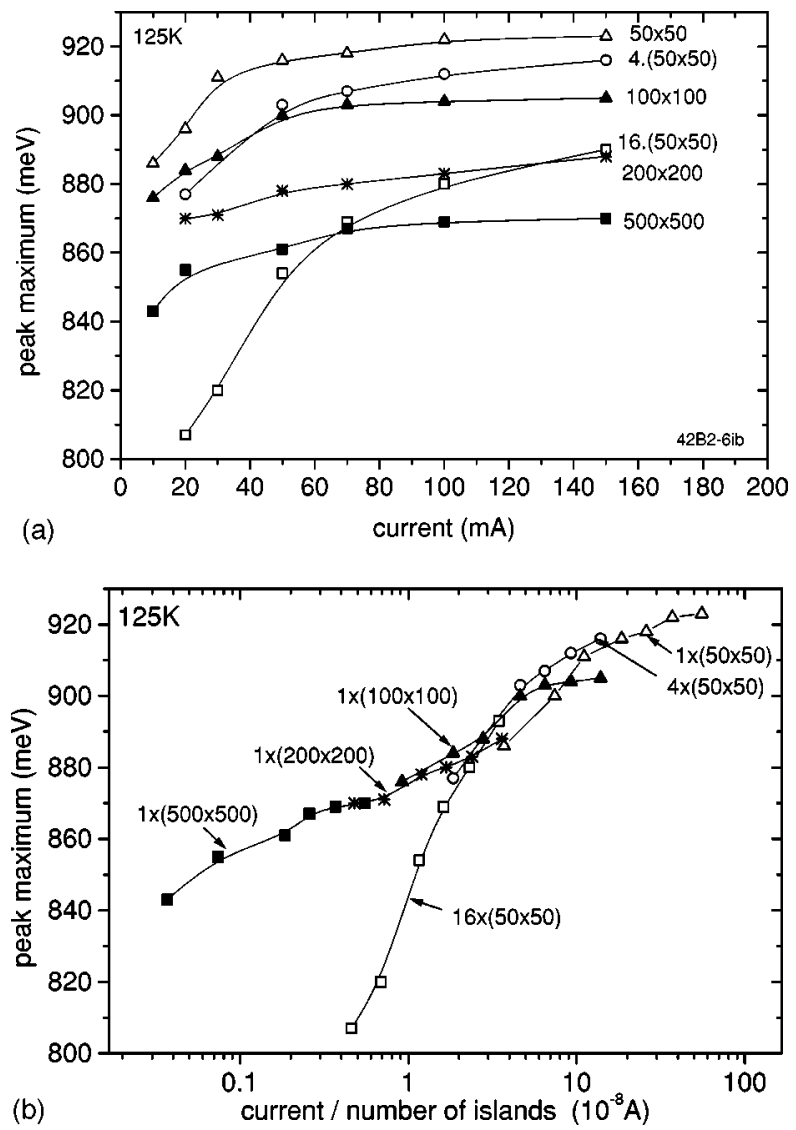

FIG. 8. Shift of the EL peak maximum with (a) current and (b) with current divided by the number of islands in a diode.

currents and the different behavior between small and large diodes remains. Another aspect is the current through the facets, whose contribution increases by decreasing the diode area, as discussed in Sec. III. Figure 3(a) shows that the current through facets increases the total current by approximately, 10 times at $0.25 \mathrm{~V}$, but at higher voltages, as seen in Fig. 3(b) this "loss" of current through the facets is diminished. However, as the electroluminescence was measured at currents $\geqslant 10 \mathrm{~mA}$, the influence of current loss through facets can be neglected. Therefore, the current through facets cannot be responsible for the different behavior of the EL peak of the large and small diodes in Fig. 8(b). Another cause could be the different microstructure of the islands in large and small mesas.

The interpretation is not straightforward owing to the possible influence of the mesa size on the strain state and the Si content of Ge islands of uncapped and capped islands. These are still matters of research and debate. It is obvious that the formation of 3D islands allows the reduction of strain energy in the Ge layer and therefore the islands are at least partially relaxed. ${ }^{18,29-32}$ From the measurement of inplane strain in coherent Ge islands on $\mathrm{Si}(001)$ by TEM after deposition of $11 \mathrm{ML}$ Ge at $600^{\circ} \mathrm{C}$ by CVD using $\mathrm{GeH}_{4}$ it was deduced that no significant in-diffusion of $\mathrm{Si}$ has occured and that a strain relaxation of the island of up to $84 \%$ has taken place. ${ }^{30}$ Other authors report of a $\mathrm{Si}$ content of $40 \%$ in capped islands deposited at $750{ }^{\circ} \mathrm{C}^{33}$
Now, we try to explain the lowest values of peak position in Fig. 8(b), the value of $840 \mathrm{meV}$ for the large diodes and $807 \mathrm{meV}$ for the small diodes. These values represent upper limits for the ground state transition energy. Assuming that the difference in these energies is entirely due to a different content of $\mathrm{Si}$ in large and small diodes we evaluated the EL peak position with the following assumptions: (1) the islands are completely relaxed, (2) the island height is $\geqslant 10$ $\mathrm{nm}$ (we neglect pyramids, as their density is lower than $10 \%$ in all mesas), and (3) the band offset at conduction band is zero. For the effective mass of holes for unstrained $\mathrm{Si}_{1-x} \mathrm{Ge}_{x}$ we used the formula ${ }^{34} m_{h h}=0.2907-0.09021 x$ $+0.00552 x^{2}$. Other parameters are: Band gap of $\mathrm{Si}$ at $125 \mathrm{~K}$ : $1153 \mathrm{meV}$, and band gap of relaxed $\mathrm{Si}_{1-x} \mathrm{Ge}_{x}$ from Franz et al. ${ }^{35}$ The peak position is defined as the sum of band gap in the island and the confinement energy of holes for different $\mathrm{Ge}$ concentrations. The binding energy of excitons was neglected. The result is that the peak energy of $807 \mathrm{meV}$ corresponds to a Si content of $8 \%$, while for the peak energy of $840 \mathrm{meV}$ we find a Si content of $10 \%$. It results that the in-diffusion of $\mathrm{Si}$ into dome islands on large mesas is stronger than on small mesas. If we make a similar discussion assuming that there is no $\mathrm{Si}$ in-diffusion at all, but the strain state of islands depends on the mesa area we arrive to the conclusion that the domes on larger areas are stronger relaxed than the domes on small areas.

Concerning the EL intensity the general observation is that small area diodes emit 2-3 times more light. Figure 6(a) and 6 (b) illustrates this statement for $50 \times 50$ and $200 \times 200$ $\mu \mathrm{m}^{2}$ diodes. The units for the EL intensity are the same in Fig. 6(a) and 6(b). A correct comparison implies the same current density, therefore for the small diodes an array of 16 diodes was chosen. We see that small area diodes emit at least 2 times more light. As the emission is supposed to take place in the islands (because there is no signal from the wetting layer) one reason for this result is that in small area diodes the recombination on deep centers (Shockley-HallReed recombination) is lower, probably due to the lower total number of defects in the islands. One of the best diodes were single $50 \times 50 \mu \mathrm{m}^{2}$ mesas with a constant emission up to $170 \mathrm{~K}$, an activation energy of $100 \mathrm{meV}$, and an external quantum efficiency of $1.10^{-6}$ at $300 \mathrm{~K}$ and $\lambda=1.4 \mu \mathrm{m}$. The low efficiency at room temperature must be due to the loss of holes by the lateral diffusion into the wetting layer and then thermalization from the wetting layer. The density of islands was shown to be low, therefore carriers in the wetting layer have time enough to escape from the Ge layer before being trapped in another island.

\section{CONCLUSIONS}

We have investigated the electroluminescence of selfassembled Ge islands (mainly domes) as a function of injection current, temperature, and mesa area. The diodes were realized by selective epitaxy on patterned wafers with variable geometry. We have shown that deposition on areas of the order of the diffusion length of Ge adatoms influences size distribution. The distribution becomes narrower when the deposited area is reduced. Therefore, deposition on lim- 
ited areas are more suitable for applications where uniformity is required. The results evidence a correlation between island distribution and electroluminescence. Incorporation of the island layer deeper into the $\mathrm{p}-\mathrm{i}-\mathrm{n}$ junction could prevent loss of current in the facet region. Also, elimination of the wetting layer might improve the room-temperature EL efficiency.

\section{ACKNOWLEDGMENTS}

The authors are thankful to H.-P. Bochem for the SEM pictures, to M. Nonn for the lithographic masks, and to K. Schmidt for the Rutherford backscattering analysis. They are indebted to D. Dentel for a critical reading of the manuscript. The financial support from ISI-FZ (T.S.) and from the TMR Network Aapples nr. FMRXCT96-0029 (O.C.) is gratefully acknowledged.

${ }^{1}$ J. C. Campbell, in Germanium Silicon: Physics and Materials: Optoelectronics in Silicon and Germanium Silicon, edited by R. Hull and J. C. Bean (Academic, San Diego, 1999) p. 347.

${ }^{2}$ R. Apetz, L. Vescan, C. Dieker, and H. Lüth, Appl. Phys. Lett. 66, 445 (1995).

${ }^{3}$ L. Vescan, M. Goryll, K. Grimm, C. Dieker, IEEE Topical Meeting on Silicon Integrated Circuits for RF Systems, Ann Arbor, MI, February, 1998 , p. 38.

${ }^{4}$ L. Vescan and T. Stoica, in Silicon Based Optoelectronics, edited by D. C. Houghton and E. A. Fitzgerald (SPIE, Bellingham, 1999), Vol. 3630, p. 163.

${ }^{5}$ T. Stoica and L. Vescan, J. Appl. Phys. 83, 3367 (1998).

${ }^{6}$ E. Mateeva, P. Sutter, J. C. Bean, and M. G. Lagally, Appl. Phys. Lett. 71, 3233 (1997).

${ }^{7}$ L. Vescan, W. Jäger, C. Dieker, K. Schmidt, H. Lüth, in Mechanism of Heteroepitaxial Growth, edited by M. F. Chrishom, R. Hull, and L. J.

Schowalter (MRS Symposia, Pittsburgh, PA, 1992), Vol. 263, p. 23.

${ }^{8}$ V. LeThanh, V. Yam, P. Boucaud, F. Fortuna, C. Ulysse, D. Bouchier, L. Vervoort, and J.-M. Lourtioz, Phys. Rev. B 60, 5851 (1999).

${ }^{9}$ L. Vescan, J. Cryst. Growth 194, 173 (1998).

${ }^{10}$ L. Vescan, K. Grimm, M. Goryll, and B. Holländer, Mater. Sci. Eng., B 69-70, 324 (1999).

${ }^{11}$ L. Vescan, C. Dieker, A. Hartmann, and A. van der Hart, Semicond. Sci. Technol. 9, 387 (1994).
${ }^{12}$ L. Vescan, K. Grimm, and C. Dieker, J. Vac. Sci. Technol. B 16, 1549 (1998).

${ }^{13}$ C. Popescu, R. Apetz, and L. Vescan, J. Appl. Phys. 80, 5791 (1996)

${ }^{14}$ M. Goryll, L. Vescan, K. Schmidt, K. Szot, S. Mesters, and H. Lüth, Appl. Phys. Lett. 71, 410 (1997).

${ }^{15}$ M. Goryll, L. Vescan, and H. Lüth, Thin Solid Films 336, 244 (1998).

${ }^{16}$ M. Goryll, L. Vescan, and H. Lüth, in Epitaxial Growth-Principles and Applications, edited by A.-L. Barabasi, M. Krishnamurthy, F. Liu, and T. Pearsall (MRS Symposia, Pittsburgh, PA, 1989), Vol. 570, p. 205.

${ }^{17}$ Y.-W. Mo, D. E. Savage, B. S. Swartzenhuber, and M. G. Lagally, Phys. Rev. Lett. 65, 1020 (1990).

${ }^{18}$ D. J. Eaglesham and M. Cerullo, Phys. Rev. Lett. 64, 1943 (1990).

${ }^{19}$ M. Zinke-Allmang, L. C. Feldman, and M. H. Grabow, Surf. Sci. Rep. 16, 377 (1992).

${ }^{20}$ M. Tomitori, K. Watanabe, M. Kobayashi, and O. Nishikawa, Appl. Surf. Sci. 76/77, 322 (1994).

${ }^{21}$ G. Capellini, L. Di Gaspare, F. Evangelisti, and E. Palange, Appl. Phys. Lett. 70, 493 (1996)

${ }^{22}$ T. I. Kamins, E. C. Carr, R. S. Williams, and S. J. Rosner, J. Appl. Phys. 81, 211 (1997).

${ }^{23}$ G. Medeiros-Ribeiro, A. M. Bratkovski, T. I. Kamins, D. A. A. Ohlberg, and R. S. Williams, Science 279, 353 (1998).

${ }^{24}$ J. H. Zhu, K. Brunner, and G. Abstreiter, Appl. Phys. Lett. 72, 424 (1998).

${ }^{25}$ B. Rahmati, W. Jäger, H. Trinkhaus, R. Loo, and H. Lüth, Appl. Phys. A: Mater. Sci. Process. 62, 575 (1996).

${ }^{26}$ L. Kubler, D. Dentel, J. L. Bischoff, C. Ghica, C. Ulhaq-Bouillet, and J. Werckmann, Appl. Phys. Lett. 73, 1053 (1998).

${ }^{27}$ P. Sutter and M. G. Lagally, Phys. Rev. Lett. 81, 3471 (1998).

${ }^{28}$ R. Braunstein, A. R. Moor, and F. Herman, Phys. Rev. 109, 695 (1958).

${ }^{29}$ S. Christiansen, M. Albrecht, and H. P. Strunk, Comput. Mater. Sci. 7, 213 (1996).

${ }^{30}$ A. J. Steinfort, P. M. L. O. Scholte, A. Ettema, F. Tuinstra, M. Nielsen, E. Landemark, D.-M. Smilgies, R. Feidenhans, G. Falkenberg, L. Seehofer, and R. L. Johnson, Phys. Rev. Lett. 77, 2009 (1996).

${ }^{31}$ P. D. Miller, C.-P. Liu, W. L. Henstrom, J. M. Gibson, Y. Huang, P. Zhang, T. I. Kamins, D. P. Basile, and R. S. Williams, Appl. Phys. Lett. 75, 46 (1999).

${ }^{32}$ J. Tersoff and F. K. LeGoues, Phys. Rev. Lett. 72, 3570 (1994).

${ }^{33}$ D. Cherns, A. Hovsepian, and W. Jäger, J. Electron Microsc. 47, 211 (1998).

${ }^{34} \mathrm{P}$. Gartner (unpublished).

${ }^{35}$ M. Franz, K. Pressel, A. Barz, P. Dold, and K. W. Benz, J. Vac. Sci. Technol. B 16, 1717 (1998). 